\title{
Las universidades como agentes de fortalecimiento de la cultura tributaria: caso NAF de la UCACUE
}

Universities as agents for strengthening the tax culture: UCACUE NAF case

\section{Universidades como agentes de fortalecimento da cultura tributária: caso UCACUE NAF}

\author{
Patricio Mendieta-Andrade ${ }^{1}$ \\ Universidad Católica de Cuenca (Ecuador) \\ pmendietaa@ucacue.edu.ec \\ Joseline Calle-Santana ${ }^{2}$ \\ Universidad Católica de Cuenca (Ecuador) \\ jdcalles24@est.ucacue.edu.ec \\ Lorena Vintimilla-Paredes \\ Universidad Católica de Cuenca (Ecuador) \\ Irvintimillap80@est.ucacue.edu.ec \\ Claudia Riera-Ceballos \\ Universidad Católica de Cuenca (Ecuador) \\ cvrierac02@est.ucacue.edu.ec
}

Fecha de recepción: 20 de octubre de 2020

Fecha de recepción evaluador: 20 de noviembre de 2020

Fecha de recepción corrección: 20 de diciembre de 2020

\footnotetext{
${ }^{1}$ Patricio Mendieta-Andrade es Ingeniero Comercial, Magister en Gestión Tecnológica, Magister en Contabilidad y Finanzas, Docente de la Universidad Católica de Cuenca, asesor contable y tributario, investigador en el ámbito de la contabilidad y tributación. ORCID: http://orcid.org/0000-0001-9596-434

2 Joseline Calle-Santana (ORCID: https://orcid.org/0000-0002-6739-4150), Lorena Vintimilla-Paredes (ORCID: https://orcid.org/0000-0002-6520-1095) y Claudia Riera-Ceballos (ORCID: https://orcid.org/0000-0002-6644-3651) son estudiantes de la Carrera de Contabilidad de la Universidad Católica de Cuenca y empleadas privada..
} 


\title{
Resumen
}

Considerando la importancia que tiene en nuestro país la recaudación fiscal en el Presupuesto General del Estado y siendo que ésta se garantiza en el largo plazo con una cultura tributaria consolidada, en el presente documento, se exponen los principales resultados de la evaluación del impacto del Proyecto Núcleo de Apoyo Contable y Fiscal de la Universidad Católica de Cuenca, como actor proactivo, en su fortalecimiento. Para el fin propuesto se planteó una investigación del tipo cualitativa, basada en el estudio de caso, utilizando como técnica de investigación la entrevista semiestructurada sobre un grupo de doce beneficiarios del Proyecto anotado, con lo cual se evidenció las características más relevantes de su cultura tributaria y se identificó el impacto del Proyecto en el fortalecimiento de la misma.

Palabras clave: Cultura tributaria, evaluación de impacto, vinculación con la sociedad.

\begin{abstract}
Considering the importance that tax collection has in our country in the General State Budget and being that it is guaranteed in the long term with a consolidated tax culture; In this document, the main results of the evaluation of the impact of the Nucleus of Accounting and Fiscal Support Project of the Catholic University of Cuenca are exposed, as a proactive actor, in its strengthening. For the proposed purpose, a qualitative research was proposed based on the case study, using as a research technique the semi-structured interview on a group of twelve beneficiaries of the annotated Project, with which the most relevant characteristics of their tax culture and the impact of the Project in strengthening it is recognized.
\end{abstract}

Key words: Tax culture, impact evaluation, link with society.

\section{Resumo}

Considerando a importância que a cobrança de impostos no nosso país tem no Orçamento Geral do Estado e sendo que esta é garantida a longo prazo com uma cultura fiscal consolidada; Neste documento, são expostos os principais resultados da avaliação do impacto do Projeto do Núcleo de Apoio Contábil e Fiscal da Universidade Católica de Cuenca, como ator proativo, no seu fortalecimento. Para o efeito proposto, foi proposta uma pesquisa qualitativa a partir do estudo de caso, utilizando como técnica de pesquisa a entrevista semiestruturada a um grupo de doze beneficiários do Projeto anotado, com a qual as características mais relevantes da sua cultura tributária e do impacto do Projeto no fortalecimento é reconhecido.

Palavras-chave: Cultura tributária, avaliação de impacto, vínculo com a sociedade. 


\section{Introducción}

La Universidad Católica de Cuenca (UCACUE) en cumplimiento de su apostolado de servicio al pueblo, en el año 2016 puso en ejecución, en el marco del Acuerdo de Cooperación Interinstitucional con el Servicio de Rentas Internas (SRI), el Proyecto Núcleo de Apoyo Contable y Fiscal (NAF), con el objetivo de contribuir al fortalecimiento de la cultura tributaria en el cantón Cuenca; siendo uno de sus objetivos específicos el brindar asesoramiento gratuito y eficaz a los contribuyentes del cantón, apoyando de ésta manera a la reducción del incumplimiento tributario, y facilitando el acceso a los beneficios fiscales de sus beneficiarios (UCACUE, 2016). De ésta manera, la presente investigación se propone evaluar los posibles impactos del Proyecto NAF de la UCACUE en sus beneficiarios, visibilizando el aporte de las Institución en el fortalecimiento de la cultura tributaria, radicando su importancia en que los resultados de la misma se constituirán en instrumentos para la toma de decisiones, tanto en los centros de estudio como a nivel gubernamental.

El Proyecto NAF de la UCACUE se establece como un espacio en el que los estudiantes de la Unidad Académica de Administración de Empresas de dicha casa de estudios, integran la teoría con la práctica en su proceso de aprendizaje, mediante la Practicas Pre Profesionales de Servicio Comunitario, enfocándose a brindar un servicio social, ejecutándose en los últimos ciclos de las carreras con un total de ciento sesenta horas, según se especifica en el Reglamento de Vinculación con la Sociedad de la Universidad (Secretaría General, UCACUE, 2015)

En base a los archivos del Proyecto NAF de la UCACUE, desde su inicio hasta junio del 2020, participaron en el Proyecto 118 estudiantes de la Unidad Académica de Administración de Empresas, quienes realizaron 8.093 asesoramientos; prestando los siguientes servicios, los cuales se encuentran definidos por el SRI (s/f):

- Información general contable y para la presentación oportuna de obligaciones y trámites tributarios (RUC, facturación, declaraciones y anexos, vehículos, etc.)

- Información general sobre obligaciones tributarias pendientes.

- Información general sobre el Régimen Impositivo Simplificado (RISE).

- Información general sobre medios de pago de impuestos y deudas tributarias.

- Información general sobre devolución de impuestos a Adultos Mayores y Discapacitados.

- Información general para la utilización de los servicios en línea del SRI. 


\section{Conceptualización de la cultura tributaria}

El sistema tributario se institucionaliza por medio de la normativa legal, cuya difusión, aceptación y práctica es indispensable para desarrollar una cultura tributaria, concebida ésta como el conjunto de información y conocimientos que tienen los ciudadanos sobre los impuestos, el cual se integra a las percepciones, criterios, hábitos y actitudes frente a la tributación (Villasmil, M, Fandiño, Y, \& Alvarado, L, 2018).

La cultura tributaria constituye la base de un sistema tributario eficiente al largo plazo y ha sido estudiada desde su definición (Díaz, Cruz, \& Castillo, 2016), caracterización (Vanegas, 2016) y el papel que desempeñan las instituciones de educación superior en su fortalecimiento (Mendieta, P, Parra, J, \& Sánchez, G, 2020). Estos estudios reconocen tres aspectos importantes que caracterizan a la cultura tributaria de una sociedad, como son los conocimientos sobre impuestos, las actitudes de los contribuyentes frente al pago de los impuestos y la percepción del fin que se da los tributos.

En línea con lo expuesto, Reátegui (2016) destaca que la cultura tributaria se determina por dos aspectos, el uno del tipo legal, relacionado con el hecho de que el ciudadano es controlado, obligado y sancionado para el cumplimiento de los tributos y el otro de tipo ideológico, en el que se relaciona el pago de los impuestos con la satisfacción de la población sobre el uso correcto que se le da a sus impuestos y que la percepción de que al menos una parte de ellos le es retornado en forma de servicios públicos adecuados.

Se distingue que la evasión fiscal genera desajustes presupuestarios que causa ineficiencia en el aprovisionamiento de bienes y servicios públicos y socaba las políticas redistributivas; es así que Ponzano y Ottone (2019) al analizar la relación entre la "prosocialidad", entendida ésta como la preocupación por los beneficios de los demás en Italia, Reino Unido y Suecia, aun los individuos "prosociales" disminuyen su honestidad cuando el Estado es ineficiente y no se evidencia la inversión en servicios públicos, pagando de forma más consiente cuando los ingresos tributarios no son desperdiciados y su sacrificio es útil.

Confirmando lo expuesto, Barros (2013), al estudiar las motivaciones de las personas para pagar sus impuestos, destaca, entre otras variables, el indicador de retorno y la penalización legal, siendo la primera la comparación de la carga tributaria frete a los servicios gubernamentales recibidos, encontrándose que la percepción de retorno se reduce a medida que los pagos aumentan, siendo que los servicios recibidos se mantienen constantes; por otro lado, la segunda variable se explica a través la relación directa entre el temor existente de ser encontrado evadiendo impuestos y el grado de penalización que supone éste acto.

Al estudiar la disposición de las personas a pagar el impuesto a la renta en contextos geográficos distantes al nuestro, se aprecia, en el caso ucraniano, que la disposición de las personas a pagar impuestos es un componente de la cultura humana, la cual no se relaciona significativamente con el nivel de salarios y de vida, más si con 
aspectos como el nivel de comprensión de la importancia de los tributos en el desarrollo socioeconómico del Estado (Kravchenko, O, Yarmolitska, Shuliarenko, Kolumbet, \& Kharchuk, 2019). Así, en el ámbito más cercano, en el caso peruano se reconoció, entre otras, "la desconfianza sobre el acierto con que el Estado administra los recursos" y la "la emisión de normas tributarias complicadas y complejas" como causa del incumplimiento tributario en dicho país (Reátegui, 2016).

Lo expuesto, es verificado por Alasfour (2019), quien en su trabajo, al investigar la causas del incumplimiento en el pago de los tributos y las características del sistema tributario de Jordania, evidencia que los valores socioculturales son las causas más importantes de la evasión, donde la formación y funcionalidad del Estado ha creado desconfianza en los ciudadanos, recomendando, el autor, mayor transparencia y rendición de cuentas a fin de fortalecer el sistema tributario desde su base cultural.

Se reconoce que la evasión de los impuestos limita la capacidad de desarrollo económico de un Estado, por lo que es necesario establecer políticas que reflejen un gobierno responsable, transparente, eficiente, efectivo y que garantice un estado de derecho. Epstein y Gang (2019), en su trabajo, analizan las opciones que tiene la administración tributaria, dados los diferentes grupos de interés, donde los sectores ricos y pobres de la economía influyen, desde su perspectiva, en los esfuerzos realizados por el Estado para hacer cumplir el pago de impuestos.

En el caso panameño, según el estudio del Centro Interamericano de Administración Tributaria (2013), la evolución positiva en el nivel de cumplimiento tributario de los últimos años, se vería explicada, a más del control ejercido por la autoridad recaudadora, por los programas de educación implementados a fin de informar y educar a los contribuyentes sobre el pago de los impuestos como un deber cívico; siendo que, según los autores, mantener informados a los contribuyentes sobre sus obligaciones constituye un factor importante en el cumplimiento de los mimos.

Finalmente, cabe destacar el papel de la educación como uno de los pilares básicos para la construcción de la cultura tributaria de los ciudadanos, existiendo evidencia de la relación positiva entre su fortalecimiento y la calidad de la información disponible sobre lo político (Zapata, R, Rodríguez, C, \& Torres. K, 2017). En éste marco, se identifica que debido a los constantes cambios en la legislación fiscal, se crea un desconocimiento de los impuestos, generando un una baja concientización de la importancia del pago de los mismos, aun en los estudiantes de carreras universitarias de carácter contable, por lo que se recomienda fortalecer los programas de educación a fin de sensibilizar a los contribuyentes sobre la importancia del pago (Mendza, F, Palomino, R, Robles, J, \& Ramírez, S, 2016). 


\section{Metodología}

La presente investigación se define como del tipo empírica de carácter cualitativa, basada en el método del estudio de casos. Se utilizó esta metodología debido a su pertinencia para medir y registrar la conducta de las personas involucradas en el fenómeno que se estudia, mediante la utilización de la entrevista (Tola, Lozano, \& Romero, 2018).

El estudio se desarrolló en la ciudad de Cuenca-Ecuador, debido a que el mismo se enmarca en el ámbito de influencia territorial del Proyecto NAF de la UCACUE; siendo que el trabajo de campo comenzó en el año 2019 y se extendió hasta el mes de mayo de 2020, considerándose el presente como un estudio de tipo longitudinal, en el que se realizaron entrevistas del tipo semiestructuradas a 12 personas beneficiarias del Proyecto en los dos momentos de tiempo ya indicados.

Como se indicó, se utilizó como técnica de investigación la entrevista semiestructurada o entrevista etnográfica, la cual se define, según la literatura, como una conversación entre el investigador y el informante, siendo el primero quien escucha atentamente sin imponer e interpretar las respuestas, guiando únicamente el desarrollo de la entrevista con el fin de reconocer la vida cultural y social del grupo de estudio por la interpretación subjetiva que explica la conducta común del grupo (Kallio, H, Pietilä, A. M, Johnson, M, \& Kangasn, M, 2016)

La entrevista se planteó como instrumento para recopilar los datos, a fin de obtener una perspectiva amplia sobre el impacto del Proyecto en los diferentes aspectos inherentes a la cultura tributaria de los beneficiarios del mismo. El trabajo de investigación se estructuró en base a tres componentes principales, en el primero se realizaron las entrevistas semiestructuradas con los beneficiarios, utilizando un cuestionario flexible, consistente con el método inductivo de análisis de la información según la literatura pertinente (Amdrade, Alejo, \& Armendariz, 2018). Se realizaron 24 entrevistas en total, basados en las consideraciones que hace Martínez (2019), sobre las oportunidades de la aplicación de esta técnica en la investigación cualitativa. En un segundo componente de la investigación, se realizó el seguimiento de las consultas concretadas por los beneficiaros que participaron en el estudio, para lo cual se utilizó los registros del Proyecto NAF, referente a las atenciones solicitadas por los mismos. Finalmente, con los datos obtenidos se construyó una síntesis transversal de casos, para analizar la evidencia obtenida de los casos de estudio.

En el estudio se plantearon tres variables dependientes o de interés, con el fin de caracterizar aspectos inherentes a la cultura tributaria del grupo de estudio, definidas como: conocimientos de los contribuyentes sobre los impuestos, actitud frente al pago de los tributos y percepción del fin que da el Estado a los impuestos cobrados. Siendo que la variable independiente o explicativa del caso sería el impacto generado con las actividades realizadas en el Proyecto NAF de la UCACUE. 
El proceso de muestreo para la selección de los casos siguió un enfoque teórico, en el que se destaca como propósito "maximizar las oportunidades de comparar acontecimientos, incidentes o sucesos", siendo que los casos se especifican dada su respuesta al modelo teórico previo para la conceptualización del problema estudiado (Pérez, R, Lagos, L, Mardones, R, \& Sáez, F, 2017, p. 13). De esta manera, se optó por un grupo de estudio formado por 4 beneficiarios caracterizados en su actividad actual como jubilados, 4 microempresarios y 4 empleados en relación de dependencia. Los entrevistados se seleccionaron del listado de beneficiarios del Proyecto que podría contribuir con información a la investigación, procurando incluir sujetos, cuyo discurso aportara significados relevantes con relación al desarrollo de la cultura tributaria, es decir, la selección se vinculó a la búsqueda de informantes calificados que contribuyeran al logro de los fines de la investigación.

Las entrevistas se realizaron con una guía semiestructurada, la cual duró entre 20 y 50 minutos. En la Tabla 1 se presentan las principales características de los tres casos analizados, donde los nombres son omitidos para garantizar la objetividad del estudio.

Tabla 1: Perfil de los casos de estudio

\begin{tabular}{cccccc}
\hline Casos & $\begin{array}{c}\mathbf{N}^{\circ} \text { de } \\
\text { casos }\end{array}$ & $\begin{array}{c}\text { Edad } \\
\text { promedio }\end{array}$ & Actividad & $\begin{array}{c}\text { Número promedio } \\
\text { de consultas } \\
\text { presentadas en el } \\
\text { período }\end{array}$ & $\begin{array}{c}\mathbf{N}^{\circ} \text { de } \\
\text { entrevistas }\end{array}$ \\
\hline Tipo 1 & 4 & 67 & Jubilado & 10 & 8 \\
Tipo 2 & 4 & 36 & Microempresarios & 8 & 8 \\
Tipo 3 & 4 & 25 & $\begin{array}{c}\text { Empleado en relación de } \\
\text { dependencia }\end{array}$ & 4 & 8 \\
\hline
\end{tabular}

Fuente: Elaboración propia

A continuación, se presenta el resumen y síntesis transversal de los casos. Las siguientes 3 , secciones informan los resultados obtenidos de los tres casos involucrados en nuestra investigación.

\section{Resultados y discusión}

\section{Conocimientos de los contribuyentes sobre los impuestos}

En éste punto se presentan los resultados obtenidos en las entrevistas a los beneficiarios del Proyecto NAF de la UCACUE que participaron en el presente estudio, con los cuales se identificaron aspectos inherentes a los conocimientos de los contribuyentes sobre los impuestos y el impacto del Proyecto en su fortalecimiento, lo cuales se resumen en la Tabla 2. 


\section{Medios por los que se informa sobre los impuestos}

Al cuestionar a los entrevistados sobre el medio por el cual ha recibido información referente a los temas tributarios, se verifica una clara diferencia entre los tres tipos de casos planteados. Es así que, en el caso de los empleados en relación de dependencia, éstos afirmaron que conocieron sobre sus obligaciones fiscales por medio de la prensa en formato digital, principalmente.

En cuanto al grupo de los microempresarios, éstos informaron que no han tenido acceso a información de sus deberes tributarios por algún medio en específico, pues siempre han sido los profesionales de la contabilidad, que han contratado, los que les han guiado para el cumplimiento de los mismos.

Finalmente, el grupo de los jubilados, comentó que conocieron de sus beneficios tributarios por medio de sus familiares y amigos, también indicaron, en algunos casos, que han recibido información en conferencias presentadas por parte de funcionarios del SRI o estudiantes de las Universidades de la ciudad, incluidas las presentadas por los estudiantes pertenecientes al Proyecto NAF de la UCACUE, en las que se informó sobre los beneficios a los que tienen derecho.

En los tres casos coincidieron en afirmar que sus conocimientos referentes al cumplimiento de sus impuestos mejoraron considerablemente desde que asisten con regularidad a las oficinas del Proyecto NAF de la UCACUE, donde los estudiantes les asesoraron e informaron sobre sus obligaciones. Destacan la importancia de contar con los conocimientos a fin de cumplir con sus impuestos y que su cumplimiento a mejorado totalmente con el apoyo de los estudiantes del Proyecto.

\section{Conocimiento de la normativa legal tributaria}

En los tres casos estudiados, se evidencia su pleno conocimiento del hecho de que la Asamblea Nacional emite las Leyes de carácter tributario, más se desconoce en todos los casos el ordenamiento jurídico actual, siendo desconocido el Reglamento para la Aplicación de la Ley de Régimen Tributario Interno y las Resoluciones pertinentes al tema tributario ignorando su función y el ente que las emite. En ejercicio de sus funciones en el Proyecto NAF, los estudiantes dieron a conocer detalles sobre éstos últimos, refiriéndose una mejora en el conocimiento sobre el tema en los tres casos.

Por otro lado, en el caso de jubilados, estos informaron que la legislación cambia constantemente, lo cual, en ocasiones dificulta el acceso a sus beneficios tributarios. Refieren que la reducción en los montos máximos a solicitarse por concepto de devolución a partir del año 2016 afectó considerablemente su ingreso por éste beneficio. Anotan que desconocen el motivo de ésta reducción, siendo que conocían que en primera instancia se lo hizo en respuesta a un aporte a las finanzas nacionales afectadas por el terremoto de abril de dicho año; más desconocen por que persiste la reducción. 


\section{Acceso a la información y datos en temas tributarios}

Al consultar a los beneficiarios del Proyecto NAF, sobre la facilidad que estos perciben para poder acceder a su información en temas tributarios, concretamente las resoluciones emitidas por el SRI, estos, en los tres casos, refieren que se complica el acceso debido a que para acceder a la misma es necesario ingresar al Sistema Informático del SRI, lo cual, debido a su limitado conocimiento sobre el uso de las herramientas tecnológicas y el manejo del Sistema anotado, reconociendo su dificultad para obtener la información anotada.

Se les consultó si tenía acceso a su correo electrónico y si consideraban a éste como un medio accesible para conocer sobre las notificaciones del SRI; siendo que, en el caso de los microempresarios y los empleados en relación de dependencia, éstos respondieron que no tenían problema en acceder a sus correos personales y conocer sobre sus notificaciones del SRI; mas, en el caso de los jubilados, éstos supieron indicar, de forma generalizada, que no podían ingresar a su correo electrónico y que necesitaban, bien sea de un familiar o de los alumnos del Proyecto NAF, para ingresar al mismo, siendo que el uso limitado de la tecnología dificultaba el acceso a su información.

\section{Conocimiento sobre el fin que el estado da a los impuestos recaudados}

En los tres casos existe coincidencia en afirmar que conocen a plenitud, según refieren los medios de comunicación, que los impuestos que pagan se destinan a mantener los gastos necesarios para que el Estado cumpla con sus funciones, siendo principalmente referidas salud, educación, seguridad. Afirman que desconocen exactamente el porcentaje del Presupuesto General del Estado que es cubierto por la recaudación fiscal y los valores que se destinan a los gastos ya anotados. Los entrevistados afirmaron que no recibieron información sobre el tema por ningún medio, inclusive del NAF de la UCACUE.

Tabla 2. Impacto del Proyecto NAF en el conocimiento de los contribuyentes sobre los impuestos

\begin{tabular}{|c|c|c|}
\hline Casos & $\begin{array}{c}\text { Características inherentes a la cultura } \\
\text { tributaria identificadas }\end{array}$ & $\begin{array}{c}\text { Impacto identificado del } \\
\text { Proyecto NAF de la UCACUE en } \\
\text { el fortalecimiento de la cultura } \\
\text { tributaria }\end{array}$ \\
\hline Tipo 1 & $\begin{array}{l}\text { - Obtienen información sobre los } \\
\text { impuestos de familiares y amigos. } \\
\text { - Desconoce el ordenamiento jurídico } \\
\text { pertinente. } \\
\text { - Se le dificulta recibir información por } \\
\text { medio del correo electrónico. } \\
\text { - Desconoce aspectos los valores de los } \\
\text { diferentes tipos de gastos que hace el } \\
\text { Estado con sus impuestos }\end{array}$ & $\begin{array}{l}\text { - Mejora considerable del } \\
\text { conocimiento sobre el } \\
\text { cumplimiento de sus impuestos. } \\
\text { - Mejora en el conocimiento } \\
\text { referente al Reglamento para la } \\
\text { Aplicación de la Ley de } \\
\text { Régimen Tributario Interno y las } \\
\text { Resoluciones pertinentes al tema } \\
\text { tributario }\end{array}$ \\
\hline Tipo 2 & $\begin{array}{l}\text { - Obtiene información de los impuestos } \\
\text { en medios de prensa digital. }\end{array}$ & $\begin{array}{l}\text { - Mejora considerable del } \\
\text { conocimiento sobre el } \\
\text { cumplimiento de sus impuestos. }\end{array}$ \\
\hline
\end{tabular}




\begin{tabular}{|c|c|c|}
\hline & $\begin{array}{l}\text { - Desconoce el ordenamiento jurídico } \\
\text { pertinente. } \\
\text { - Accede con facilidad a notificaciones } \\
\text { del SRI por medio del correo } \\
\text { electrónico. } \\
\text { - Desconoce aspectos los valores de los } \\
\text { diferentes tipos de gastos que hace el } \\
\text { Estado con sus impuestos }\end{array}$ & $\begin{array}{l}\text { - Mejora en el conocimiento } \\
\text { referente al Reglamento para la } \\
\text { Aplicación de la Ley de } \\
\text { Régimen Tributario Interno y las } \\
\text { Resoluciones pertinentes al tema } \\
\text { tributario }\end{array}$ \\
\hline Tipo 3 & $\begin{array}{l}\text { - Recibe información sobre impuestos } \\
\text { de los contadores contratados. } \\
\text { - Desconoce el ordenamiento jurídico } \\
\text { pertinente. } \\
\text { - Accede con facilidad a notificaciones } \\
\text { del SRI por medio del correo } \\
\text { electrónico. } \\
\text { - Desconoce aspectos los valores de los } \\
\text { diferentes tipos de gastos que hace el } \\
\text { Estado con sus impuestos }\end{array}$ & $\begin{array}{l}\text { - Mejora considerable del } \\
\text { conocimiento sobre el } \\
\text { cumplimiento de sus impuestos. } \\
\text { - Mejora en el conocimiento } \\
\text { referente al Reglamento para la } \\
\text { Aplicación de la Ley de } \\
\text { Régimen Tributario Interno y las } \\
\text { Resoluciones pertinentes al tema } \\
\text { tributario }\end{array}$ \\
\hline
\end{tabular}

Fuente: Elaboración de los autores basado en los resultados de las entrevistas

\section{Actitud frente al pago de los tributos}

En esta segunda sección del presente apartado expositivo de los resultados de la investigación, se presentan los resultados de la evaluación de la actitud de los beneficiaros del Proyecto frente al pago de sus tributos; siendo que en la Tabla 3 se expone el impacto identificado del Proyecto sobre dicho aspecto.

\section{Contribución social}

En los tres casos hubo coincidencia en referir de manera enfática que el pago de los impuestos lo hacían como una forma de contribución social, mas, como se verá en el apartado siguiente, hubo contradicciones en las respuestas.

Como ya se anotó, en todos los casos los participantes del estudio desconocían cual era la participación real de los impuestos en el Presupuesto General del Estado, aspecto que fue explicado en la primera entrevista. Posteriormente, al realizar la segunda entrevista en el mes de mayo del presente año, en el marco de la emergencia sanitaria, los entrevistados reconocieron como muy importante la contribución social que hacen por medio del pago de sus impuestos y la utilidad que tienen éstos hoy en día, dada la situación que se atraviesa.

En el caso de los jubilados, estos refirieron en la primera entrevista que en realidad su pago de impuestos es mínimo, desconociendo que al realizar toda compra se paga un valor por concepto de Impuesto al Valor Agregado, lo cual fue explicado a detalle. Es así que, en la segunda entrevista estaban más conscientes del aporte que realizaban por dicho impuesto y el aporte que habían hecho durante sus años de vida y más aún en años pasados cuando eran parte más activa del aparato productivo nacional. 


\section{Temor a multas y sanciones}

En la primera ronda de entrevistas, luego de anotadas las respuestas al considerando anterior, se consultó si el pago de los impuestos lo hacían por temor a las multas y sanciones, lo cual causó una reacción de reconsideración, pues en todos los casos a excepción de los jubilados, se evidenció la existencia de un temor a ser objeto de sanciones por parte del SRI.

En el caso de los microempresarios, fueron explícitos en indicar que ya habían sido objeto de multas, básicamente, por presentación tardía de sus declaraciones de impuestos y en un caso se indicó que su local comercial había sido sancionado con la clausura por la falta de entrega de documentos de venta autorizados. Es así que, las cuatro personas de éste grupo de estudio, dudaron al momento de decidirse por su motivación para pagar sus impuestos; siendo que, tendían más al pago de los tributos como una obligación, en perspectiva de evitar el pago de multas y sanciones con las correspondientes pérdidas en sus negocios.

En el caso de los microempresarios beneficiarios del Proyecto NAF de la UCACUE, se evidenció, en los registros del mismo, que durante el período de estudio asistieron de forma regular a las oficinas del Proyecto para recibir la guía para la presentación de sus declaraciones; siendo que, en la segunda ronda de entrevistas, éstos refirieron que el servicio que brinda el Proyecto es provechoso a fin de cumplir a tiempo con sus tributos y les ha evitado el pago de multas.

En el caso del grupo de los empleados por dependencia, al sugerir la posibilidad de que su pago de impuestos lo harían para evitar sanciones, fueron todos contundentes en anotar que en algún momento tuvieron que pagar multas por presentación tardía de sus declaraciones de impuestos. Es así que, aunque muchas veces no generaban impuesto, principalmente en el caso del impuesto a la renta, refirieron que, con el apoyo del Proyecto, este año, concretamente, no pagaron multas, más conocieron que tenían la posibilidad de solicitar la devolución de los valores retenidos en el año 2019 por concepto de impuesto a la renta, beneficio tributario que desconocían.

Cabe anotar que, según se evidencia en los registros del Proyecto, los beneficiarios catalogados como empleados, si bien tuvieron ingresos que sobrepasaron la Fracción Básica Imponible para el Impuesto a la Renta, presentaron un nivel de gastos personales que hizo que su Base Imponible Gravada sea reducida y no cause impuesto; mas, según la legislación vigente, tiene la obligación de presentar su declaración anual de Impuesto a la Renta y el correspondiente Anexo de Gastos Personales, de lo cual refirieron que no tenían conocimiento y que lo encontraban confuso; más con el apoyo y explicación de los estudiantes del Proyecto ahora tienen claro el tema y han evitado el pago de multas, como en años anteriores.

En cuanto al grupo de jubilados refirieron que ellos no tienen la obligación de hacer declaraciones de impuestos por cuanto están ya exentos del riesgo de incurrir incumplimientos y por ende ser sancionados o multados. 
Tabla 3. Impacto del Proyecto NAF en la actitud de sus beneficiarios frente al pago de los tributos

\begin{tabular}{|c|c|c|}
\hline Casos & $\begin{array}{c}\text { Características inherentes a la cultura } \\
\text { tributaria identificadas }\end{array}$ & $\begin{array}{c}\text { Impacto identificado del Proyecto } \\
\text { NAF de la UCACUE en el } \\
\text { fortalecimiento de la cultura } \\
\text { tributaria }\end{array}$ \\
\hline Tipo 1 & $\begin{array}{l}\text { - Descimiento total de la participación } \\
\text { real de los impuestos en el } \\
\text { Presupuesto General del Estado. } \\
\text { - No identifican al pago el IVA en } \\
\text { toda compra como un impuesto. }\end{array}$ & $\begin{array}{l}\text { - Mejora en el conocimiento sobre } \\
\text { la importante participación de los } \\
\text { impuestos en el Presupuesto } \\
\text { General del Estado. } \\
\text { - Reconocimiento del aporte que } \\
\text { hacen con el pago del IVA en las } \\
\text { transacciones. }\end{array}$ \\
\hline Tipo 2 & $\begin{array}{l}\text { - Descimiento total de la participación } \\
\text { real de los impuestos en el } \\
\text { Presupuesto General del Estado. } \\
\text { - Alto temor a multas y sanciones por } \\
\text { parte del SRI por experiencias } \\
\text { pasadas, principalmente temor a } \\
\text { clausuras. }\end{array}$ & $\begin{array}{l}\text { - Mejora en el conocimiento sobre } \\
\text { la importante participación de los } \\
\text { impuestos en el Presupuesto } \\
\text { General del Estado. } \\
\text { - Mejora en el cumplimiento de } \\
\text { los impuestos y reducción de } \\
\text { multas. }\end{array}$ \\
\hline Tipo 3 & 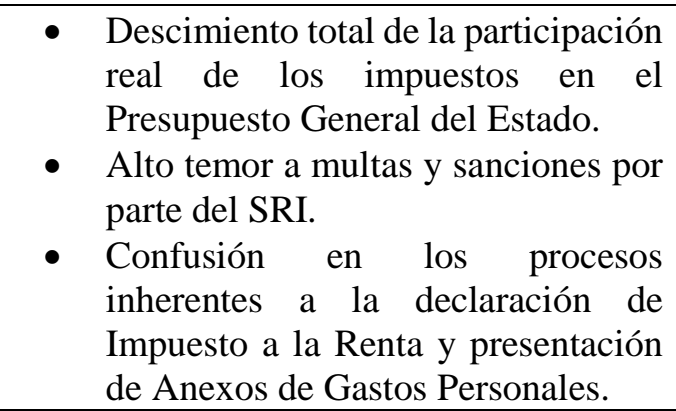 & $\begin{array}{l}\text { - Mejora en el conocimiento sobre } \\
\text { la importante participación de los } \\
\text { impuestos en el Presupuesto } \\
\text { General del Estado. } \\
\text { - Reducción en el pago de multas } \\
\text { por presentación tardía de } \\
\text { declaraciones y anexos. }\end{array}$ \\
\hline
\end{tabular}

Fuente: Elaboración de los autores basado en los resultados de las entrevistas

\section{Percepción del fin que da el estado a los impuestos cobrados}

En este punto se presentan las señas particulares que reflejó el estudio planteado, sobre las percepciones de los participantes sobre el fin que el Estado da a los impuestos cobrados por concepto de impuestos, los cuales se sintetizan en la Tabla 4.

\section{Transparencia de la información}

En todos los casos se refiere que, en medios de comunicación televisiva y periódica tanto electrónica como física, funcionarios del SRI han informado sobre altos valores recaudados por concepto de impuestos, indicando, en años pasados, que éstos han estado constantemente en crecimiento.

En esta pregunta, los entrevistados confirman lo ya anotado, en cuanto a desconocer los valores de los gastos del Estado que son cubiertos por los impuestos que pagan. 


\section{Percepción del buen uso que se da a los tributos pagados}

En la primera ronda de entrevistas, es decir en el año 2019, los participantes, en todos los casos, coincidían en reconocer que tienen una mala percepción del buen uso que se da a los tributos por parte del Estado; pues en el grupo de los microempresarios refieren, tres personas de las cuatro entrevistadas, que consideran que existen muchos gastos innecesarios, principalmente en empleados públicos.

En el caso de los jubilados todos respondieron que, en su experiencia a través de sus años de vida, han podido evidenciar diferentes casos de despilfarro y actos de corrupción, que han afectado al uso adecuado del dinero aportado por concepto de impuestos. Así también, reconocen que es evidente la inversión que hace el Gobierno en obras para bien de la sociedad, principalmente, carreteras y hospitales.

De la misma manera al aplicar la segunda entrevista en el mes de mayo del presente año, las respuestas fueron contundentes, al indicar que se ha puesto en evidencia el mal uso, en muchos casos, del dinero del estado proveniente de los impuestos, pues se han denunciado diferentes casos de uso doloso de estos fondos, siendo que se evidencia un deterioro en el indicador de retorno percibido por los entrevistados; es decir que no se aprecia un retorno de sus aportes a través de los servicios que brinda el Estado.

Con los antecedentes presentados, todos los entrevistados concluyeron que tiene una mala percepción del buen uso que se da a los tributos, siendo identificado un sentimiento de desconfianza por parte de los contribuyentes frente al fin que el Gobierno da sus aportes. Al indagar el roll del Proyecto NAF en la percepción anotada, se reconoce que no ha habido mayor aporte por cuanto, los entrevistados, explican que su percepción se basa en la información que reciben, principalmente de los medios de comunicación y no han recibido información al respecto por parte del Proyecto.

Tabla 4. Impacto del Proyecto NAF en la percepción del fin que da el Estado a los impuestos cobrados

\begin{tabular}{|c|c|c|}
\hline Casos & $\begin{array}{c}\text { Características inherentes a la cultura tributaria } \\
\text { identificadas }\end{array}$ & $\begin{array}{c}\text { Impacto identificado del } \\
\text { Proyecto NAF de la UCACUE } \\
\text { en el fortalecimiento de la } \\
\text { cultura tributaria }\end{array}$ \\
\hline Tipo 1 & $\begin{array}{l}\text { - Reconocen altos valores de recaudación de } \\
\text { impuestos por parte del SRI. } \\
\text { - Identifican un constantemente crecimiento de la } \\
\text { recaudación por concepto de impuestos en los } \\
\text { últimos años. } \\
\text { - Identifican casos de despilfarro y actos de } \\
\text { corrupción en el uso de los impuestos. } \\
\text { - Reconocen la inversión de los impuestos en obras } \\
\text { principalmente en carreteras y hospitales. } \\
\text { - En el año 2020 se acentúa la percepción del mal } \\
\text { uso de los tributos debido a las últimas denuncias } \\
\text { de usos dolosos de los mismos. }\end{array}$ & $\begin{array}{l}\text { - No se evidencia impacto } \\
\text { del Proyecto NAF en la } \\
\text { percepción del fin que da } \\
\text { el Estado a los impuestos } \\
\text { cobrados. }\end{array}$ \\
\hline Tipo 2 & $\begin{array}{l}\text { - Reconocen altos valores de recaudación de } \\
\text { impuestos por parte del SRI. }\end{array}$ & $\begin{array}{l}\text { - No se evidencia impacto } \\
\text { del Proyecto NAF en la }\end{array}$ \\
\hline
\end{tabular}




\begin{tabular}{|c|c|c|}
\hline & $\begin{array}{l}\text { - Identifican un constantemente crecimiento de la } \\
\text { recaudación por concepto de impuestos en los } \\
\text { últimos años. } \\
\text { - Reconocen alto gasto innecesario principalmente } \\
\text { en burocracia. } \\
\text { - En el año } 2020 \text { se acentúa la percepción del mal } \\
\text { uso de los tributos debido a las últimas denuncias } \\
\text { de usos dolosos de los mismos. }\end{array}$ & $\begin{array}{l}\text { percepción del fin que da } \\
\text { el Estado a los impuestos } \\
\text { cobrados. }\end{array}$ \\
\hline Tipo 3 & $\begin{array}{l}\text { - Reconocen altos valores de recaudación de } \\
\text { impuestos por parte del SRI. } \\
\text { - Identifican un constantemente crecimiento de la } \\
\text { recaudación por concepto de impuestos en los } \\
\text { últimos años. } \\
\text { - En el año } 2020 \text { se acentúa la percepción del mal } \\
\text { uso de los tributos debido a las últimas denuncias } \\
\text { de usos dolosos de los mismos. }\end{array}$ & $\begin{array}{l}\text { - No se evidencia impacto } \\
\text { del Proyecto NAF en la } \\
\text { percepción del fin que da } \\
\text { el Estado a los impuestos } \\
\text { cobrados. }\end{array}$ \\
\hline
\end{tabular}

Fuente: Elaboración de los autores basado en los resultados de las entrevistas

A manera de discusión se apunta que los casos estudiados muestran diferentes rasgos de la cultura tributaria en los beneficiarios del Proyecto NAF de la UCACUE; siendo que se destaca un aspecto que ha sido limitadamente estudiado, como es la tributación en las personas adultas mayores; donde se evidencia la dificultad que éstas tiene para acceder a la información concerniente a los tributos, dado que ésta se transmite, principalmente, por medios digitales, refiriéndose dificultades para el manejo de las herramientas informáticas pertinentes. De la misma manera, éste grupo de beneficiarios no se auto identifican como contribuyentes activos de impuestos; siendo que, no reconocen el aporte que hacen mediante el pago del IVA en sus compras, y la importancia de éste impuesto en el total de recaudo estatal por tributos.

Se evidencia en los casos estudiados un bajo nivel de comprensión de la importancia de los tributos en el desarrollo socioeconómico del Estado, aspecto que según los autores KravchenKo et al. (2019) afectaría la disposición de los contribuyentes a cumplir con sus impuestos.

Otro aspecto identificado en la cultura tributaria del grupo de estudio es la desconfianza del mismo, sobre el acierto con que el Estado administra los recursos que recauda, aspecto que, según los apuntes citados de Reátegui (2016), se considerarían como una causa probable del incumplimiento tributario.

Por el presente trabajo se pone en evidencia como una característica de la cultura tributaria de los beneficiarios del Proyecto NAF, que éstos cumplen con sus impuestos más por el temor a multas y sanciones que motivados por una conciencia de aporte social. Siendo así, no existiría una base sólida para una consolidación del Sistema Tributario Nacional, por lo que serían pertinentes las recomendaciones de Alasfour (2019), en el sentido de mejorar la transparencia y rendición de cuentas por parte del Estado, a fin de fortalecer dicho Sistema. 
Por el lado la educación de los contribuyentes como aspecto fortalecedor de la cultura tributaria, se reconoce como rasgo de la cultura tributaria que los contribuyentes entrevistados, refieren falta de conocimiento en el ordenamiento jurídico referente a los tributos. En cuanto a la información que reciben los contribuyentes, se identifica que esta es incompleta; pues, no conocen a plenitud el fin que el Estado da a los tributos y la importancia de éstos en las finanzas nacionales.

Finalmente, con respecto al impacto del Proyecto NAF en el fortalecimiento de la cultura tributaria de sus beneficiarios, por el presente trabajo se visibiliza su aporte como centro de difusión del conocimiento inherente al cumplimiento de los impuestos, siendo reconocido su impacto en los beneficiarios del mismo, principalmente en la consolidación de la educación en temas tributarios.

\section{Conclusiones}

En respuesta al objetivo del presente trabajo, de identificar el impacto del Proyecto NAF en el fortalecimiento de la cultura tributaria de sus beneficiarios, se evidencia, en primer lugar, diferentes rasgos característicos de la mima, en los tres diferentes tipos de casos analizados, desde las tres perspectivas que especificó la revisión bibliográfica del estudio, como son: conocimientos de los contribuyentes sobre los impuestos, actitud de éstos frente al pago de los tributos y la percepción del fin que da el Estado a los impuestos cobrados.

Es así que los contribuyentes se informan sobre el tema tributario por los medios digitales, principalmente; siendo en el caso de los jubilados por familiares y amigos, debido a su limitado uso de las herramientas tecnológicas. Es así, que se verifica el papel del Proyecto NAF en el fortalecimiento de la cultura tributara de sus beneficiarios, reconociéndose su aporte dirigido a mejorar los conocimientos de sus beneficiarios sobre el pago de los impuestos y el acceso a los beneficios tributarios; aunque no se evidencia un aporte claro a fin de socializar la participación e importancia de los impuestos para solventar los gastos del Estados y más aún los montos y conceptos a los cuales se destinó el gasto público.

En cuanto a la actitud, del grupo estudiado, frente al pago de los impuestos se identificó que éstos lo hacen, principalmente, por temor a las multas y sanciones, siendo que al no tener conocimiento pleno del papel importante que tienen sus impuestos en el Presupuesto General del Estado, no reconocen su contribución social como contribuyentes. En este caso, se identifica el impacto del Proyecto NAF en sus beneficiarios, dado que se ha logrado mejorar el conocimiento de éstos sobre la importancia de los impuestos para el cumplimiento efectivo de las funciones estatales; también, se ha verifica la contribución del Proyecto en la reducción de las multas y sanciones por parte de los contribuyentes que participan en el mismo, mediante asesorías que fomenten el cumplimiento cabal de sus obligaciones fiscales.

En cuanto a la percepción del fin que el Estado da a los valores cobrados por impuestos se destaca que es común, en todos los casos, el desconocimiento de los detalles 
sobre el uso de los impuestos; siendo importante destacar que las denuncias presentadas sobre el mal uso de los fondos públicos, por parte de algunos funcionarios en principios de este año, han fomentado la desconfianza en el Sistema Tributario, lo cual debilita la cultura tributaria de los contribuyentes y se constituye en una barrera para el fomento del pago voluntario de los impuestos. En este sentido, se identifica que el Proyecto NAF no tiene un impacto sobre la percepción del fin que da el Estado a los impuestos cobrados, dado que ésta se enmarca en la información que el contribuyente accede directamente por diferentes fuentes; siendo que, actualmente la imagen del Estado como administrador de los recursos públicos, se encuentra afectada por diferentes denuncias de malos manejos, siendo un aspecto que escapa a la esfera de acción del Proyecto.

Con los antecedentes presentados se concluye que las Universidades están en el deber social de constituirse como agentes proactivos en el fortalecimiento de la cultura tributaria de los contribuyentes, por medio de la ejecución de proyectos de vinculación con la sociedad que respondan a éste objetivo y que desde los cuales se ratifique el papel fundamental de las Instituciones de Educación Superior como agentes difusores del conocimiento y el desarrollo socioeconómico de un pueblo.

Finalmente, se reconoce las limitaciones que representa la metodología de investigación utilizada en el presente estudio, debido, principalmente, al reducido número de elementos de estudio, más los resultados de los mismos se constituyen en una aproximación a la problemática planteada, siendo un aporte para futuros estudios en los que se profundice sobre las causas de los hallazgos encontrados y se determinen posibles soluciones a los mimos.

\section{Declaración de conflictos de intereses}

La presente investigación se enmarca en los resultados obtenidos en el Proyecto Núcleo de Apoyo Contable y Fiscal de la Universidad Católica de Cuenca, Institución a la cual se deben sus autores en su calidad de docente investigador y estudiantes de la Unidad Académica de Administración, en el marco del cumplimiento de sus Prácticas Pre Profesionales de Servicio a la Comunidad.

\section{Bibliografía}

Alasfour, F. (2019). Costs of Distrust: The Virtuous Cycle of Tax Compliance in Jordan. Journal of Business Ethics, pp. 243-258. doi:https://doi.org/10.1007/s10551-0173473-y

Amdrade, F., Alejo, O., \& Armendariz, C. (2018). Método inductivo y su refutación deductista. Conrado, 14(63), 117-122. Retrieved from http://scielo.sld.cu/scielo.php?script=sci_arttext\&pid=S199086442018000300117 
Barros, B. (2013). ¿POR QUÉ LAS PERSONAS PAGAN SUS IMPUESTOS? Subjetividad y Procesos Cognitivos, 17(2), pp. 37-47. Retrieved from https://www.redalyc.org/pdf/3396/339630262002.pdf

Centro Interamericano de Administración Tributaria. (2013). Principales determinantes sociales del incumplimiento tributario de Panamá. Retrieved from https://www.ciat.org/Biblioteca/AsistenciaTecnica/Espanol/2013_incumplimient o_tributario_panama_itc_ciat-giz.pdf

Díaz, J., Cruz, B., \& Castillo, N. (2016). Cultura tributaria. Revista Publicando, 3(9), pp. 697-705.

Retrieved

from https://dialnet.unirioja.es/servlet/articulo?codigo=5879392

Epstein, G, \& Gang, I. (2019). Inequality, good governance, and endemic corruption. International Tax and Public Finance, 26(5), pp. 999-1017. Retrieved from https://link.springer.com/article/10.1007/s10797-019-09542-z

Kallio, H , Pietilä, A. M, Johnson, M, \& Kangasn, M. (2016). Systematic methodological review: developing a framework for a qualitative semi-structured interview guide. Journal of advanced nursing, 72(12), pp. 2954-2965. doi:https://doi.org/10.33064/41crscsh1203

Kravchenko, O, Yarmolitska, O., Shuliarenko, S., Kolumbet, O., \& Kharchuk, O. (2019). Scenario Modeling of Change of the Level of Tax Culture: The Case of Ukraine. Academy of Accounting and Financial Studies Journal, 23(6), p. 485. Retrieved from https://www.abacademies.org/articles/scenario-modeling-of-change-of-thelevel-of-tax-culture-the-case-of-ukraine-8769.html

Martinez, K. (2019). La entrevista semi-estructurada y las fallas en la estructura. La revisión del método desde una psicología crítica y como una crítica a la psicología. Caleidoscopio-Revista Semestral de Ciencias Sociales y Humanidades, 41, pp. 65-91. doi:https://doi.org/10.33064/41crscsh1203

Mendieta, P, Parra, J, \& Sánchez, G. (2020). El impacto de las prácticas pre profesionales en la cultura tributaria de los estudiantes de la Universidad Católica de Cuenca. Boletín de coyuntura(24), 3 31-39. doi:http://dx.doi.org/10.31164/bcoyu.24.2020.885

Mendza, F, Palomino, R, Robles, J, \& Ramírez, S. (2016). Correlación Entre Cultura Tributaria Y Educación Tributaria Universitaria: Caso Universidad Estatal De Sonora. Revista Global de Negocios, 4(1), pp. 61-76. Retrieved from https://papers.ssrn.com/sol3/papers.cfm?abstract_id=2659374

Pérez, R, Lagos, L, Mardones, R, \& Sáez, F. (2017). Pérez-Luco Arenas, R., Lagos Gutiérrez, L., Mardones Barrera, R., \& Sáez Ardura, F. (2017). Taxonomía de diseños y muestreo en investigación cualitativa. Un intento de síntesis entre las aproximaciones teórica y emergente. Ámbitos. Revista Internacional de 
Comunicación, $\quad$ pp. 1-18. $\quad$ Retrieved from https://idus.us.es/bitstream/handle/11441/68886/11.\%20institucional.us.esTaxonom\%c3\%ada\%20de\%20dise \%c3\%b1os\%20y\%20muestreo\%20en\%20inv estigaci\%c3\%b3n\%20cualitativa\%20Un\%20intento\%20de\%20s\%c3\%adntesis $\% 20$ entre\%20las\%20aproxim.pdf?sequence=1\&isAllowed $=y$

Ponzano, F, \& Ottone, S. (2019). Prosociality and fiscal honesty: Tax evasion in Italy, United Kingdom, and Sweden. In Dishonesty in Behavioral Economics (pp. 289318). doi:https://doi.org/10.1016/B978-0-12-815857-9.00016-9

Reátegui, A. (2016). Importancia de la cultura tributaria en el Perú. Revista de Investigación de Contabilidad Accounting power for business, 1(1). Retrieved from https://revistas.upeu.edu.pe/index.php/ri_apfb/article/view/898

Secretaría General, UCACUE. (2015). Reglamente de Prácticas Pre Profesionales. Retrieved from https://documentacion.ucacue.edu.ec/items/show/665

SRI. (s/f). NÚCLEOS DE APOYO CONTABLE Y FISCAL - INSTITUCIONES DE EDUCACIÓN $\quad$ SUPERIOR. $\quad$ Retrieved from https://www.sri.gob.ec/web/guest/nucleos-de-apoyo-contable-y-fiscal-naf1

Tola, M., Lozano, L., \& Romero, S. (2018). El Estudio de caso como metodología para el desarrollo de la Inteligencia Hermeneutica-Pragmatica en Ciencias Administrativas. RECIMUNDO: Revista Científica de la Investigación y el Conocimiento, 2(1), pp. 362-373. Retrieved from https://dialnet.unirioja.es/servlet/articulo?codigo $=6732886$

UCACUE. (2016). Acuerdo de cooperación interinstitucional entre la UCACUE y el SRI. [Acta convenio]. Archivos UCAUCE.

Vanegas, Y. (2016). Caracterización de cultura tributaria en estudiantes de grado noveno. Revista vinculos, $13(2)$, pp. 88-99. Retrieved from https://go.gale.com/ps/i.do?id=GALE\%7CA568975186\&sid=googleScholar\&v $=2.1 \& \mathrm{it}=\mathrm{r} \&$ linkaccess $=\mathrm{abs} \& \mathrm{issn}=1794211 \mathrm{X} \& \mathrm{p}=\mathrm{IFME} \& \mathrm{sw}=\mathrm{w}$

Villasmil, M, Fandiño, Y, \& Alvarado, L. (2018). Cultura tributaria en la educación: Un estudio. Revista de Ciencias Humanas y Sociales, 34(18), pp. 1620-1652. Retrieved from https://produccioncientificaluz.org/index.php/opcion/article/view/23988

Zapata, R, Rodríguez, C, \& Torres. K. (2017). a cultura tributaria y su incidencia en la recaudación de los tributos en el Cantón Babahoyo, Provincia de los Ríos. Dominio de las Ciencias, 3(3), pp. 45-68. Retrieved from https://dialnet.unirioja.es/servlet/articulo?codigo $=6326643$ 